clusion that valerianate of atropia had a very special and direct effect upon it, diminishing its inherent capacity for reflex phenomena. The almost specific effect of belladonna in preventing nocturnal seminal emissions is also probably due to this action.

I think, then, the conclusion is justified that, by its action apon the pneumogastric and sympathetic nerves, and also upon the medulla oblongata, atropia relieves, and ultimately cures, the neurosis called whooping-cough; and that in those cases where, from idiosyncrasy or easily-excited sympathetic action, the intensity and severity of the reflex phenomena are greatest, the beneficial action of atropia will be more marked.

Liverpool.

\section{SPREADING GANGRENOUS CELLULITIS.}

\section{BY P. BLAIKIE SMITH, M.D.}

PHYSICIAN, ABERDEEN GENERAL DISPENSARY ; LATE RESIDENT PHYSICIAN'S ASSISTANT, MIDDLESEX HOSPITAL.

THE perusal of two interesting papers upon Spreading Gangrenous Cellulitis, recently published in the columns of a medical periodical, induces me to narrate a severe case of this formidable disease which came under my care some time ago.

Towards the end of March, 1877, I was in attendance on a gentleman suffering from delirium tremens. He was upwards of forty years of age, of large and stout build, and for many years had led a most intemperate life. From past experience of this patient, I had learnt that nothing was of such service to him, when suffering from delirium tremens, as large doses of morphia injected under the skin; and accordingly this remedy was freely used for a day or two, with the result of alleviating all his symptoms.

On March 23rd he complained that when he got up to pass water it came with difficulty-a symptom which was hard to understand, as a careful examination of his urinary organs failed to elicit any cause for its occurrence. The only theory I could entertain was, that the morphia which had been administered was exhibiting in the person of my patient one of its physiological actions. Next day, however, threw more light on the matter, for he now began to complain of pain near the right verge of the anus, where the skin was found on examination to be swollen, hard, slightly red, and tender-a state of matters which led me to suspect a different cause for his difficult micturition. My suspicions were amply confirmed on March 25th, on which day the swelling increased largely, and was accompanied by much pain, radiating to the coccyx and loins. Defecation, as well as micturition, was now both difficult and painful. The temperature reached $102 \cdot 4^{\circ}$. Three days afterwards the swelling had involved the whole of the perineum to such an extent that the anus could not be seen. The skin was for the most part of a livid hue, hard and unvielding to the touch, with vesicles on the surface here and there; but in the right ischio-rectal region the lividity had deepened into a darker colour, and the unyielding feeling had given place to a crepitating softness. In a word, gangrene was present. Free incisions accordingly were made on both sides of the perineum, resulting in an enormous escape of gangrenous débris, attended by an intolerable fetor from the wound on the right side. The finger passed into this wound discovered a cavity so large that its size could not be made out exactly. The cellular tissue on the left side seemed little affected, and the rectum was free from disease.

From this date onwards (March 28th), every effort was directed to support the patient's strength, and promote the separation of the sloughs, by stimulants, opium, suitable nourishment, and antiseptic injections; but the spreading nature of the affection was not to be gainsaid. On March 29th both sides of the scrotum were attacked, and incisions therein gave vent to much gangrenous matter. By April 2nd, the skin covering the right lumbar, right iliac, and suprapubic regions of the abdomen had become livid and emphysematous, and a sloughing sore made its appearance at the upper and inner aspect of the right thigh, two inches below Poupart's ligament. The fetor arising from the sloughs was now almost overpowering, filling the whole house, nor did disinfectants, though used lavishly, seem to have much effect in abating the evil.

Little more remains to be told. The cellulitis of the abdominal wall continued to spread, and with its extension my patient's strength gradually failed, till, on April 4th, he died exhausted in the struggle with this most loathsome disease.

The case just recorded is similar in many ways to those narrated by Furneaux Jordan and Mr. Jackson. The disease occurred in an intemperate stout man of middle age, who (and this I have not mentioned before) had twice during the last ten years of his life suffered from perineal abscess, the second attack of which had left in the right ischio-rectal fossa a small sinus, which discharged a little pus from time to time. Is it possible that this vulnerable spot in the perineum induced what Mr. Jordan so aptly calls an "explosion" of the disease in that situation in preference to any other? I think so ; and will be interested to hear if others have noted a similar connexion in any case of spreading gangrenous cellulitis which may have come under their notice. Throughout the progress of the case the rectum and urethra were unaffected save by pressure, and the urine was free from albumen.

Aberdeen.

\section{NOTE ON DIPHTHERIA-DIFFUSION.}

\section{By ARTHUR DOWNES, M.D., S.Sc. CERT. CAMb.}

From investigation of numerous outbreaks of diphtheria in distinct localities, I have long been led to the conclusion that the dissemination of this disease in rural districts is mainly dependent on the numerous mild unrecognised cases, which may generally be discovered about the neighbourhood to which attention has been directed by an appearance of the disease in its severer form. In this dissemination some day-school usually plays a prominent part.

Others notice the existence of these slight cases in in. fected localities, but have regarded them as primarily noninfectious, acquiring specific diphtheritic infectivity by transmission through successive subjects. I am not aware in what way they consider that these "non-infectious" sorethroats are in the first instance transmitted from person to person so as to acquire this quality of specific infectivity; my own experience, indeed, is that they are simply cases of true diphtheria, so mild in degree that the symptoms, both local and general, are in abeyance, and pass almost unnoticed. The proof of their true character is that under suitable conditions their contagium gives rise to typical diphtheria, and, on the other hand, that they themselves may often be traced to an antecedent case of unmistakable diphtheria. They are decidedly infectious; not so highly infectious, perhaps, in a relative sense, as are the typical and malignant forms, but in the aggregate far more to be dreaded than these as foci of diffusion. As a rule, no medical advice is sought by the patient, who goes about, or attends school, as usual. In sickness returns, however, 1 have seen these cases returned not only as "sore-throat," but also as "bronchitis," "cold," \&c., and, indeed, they are, I believe, per se, and without a knowledge of their history, not specifically diagnosable. They generally far outnumber the well-marked typical cases.

A chief feature of diphtheria, noted by most observers, is its association with cold and damp. Damp, whether arising from defects of subsoil, surroundings, or of house construction, is a constant characteristic of those endemic haunts of diphtheria with which I am acquainted. Without prolonging this paper by a discussion of the various views that have been put forward as to the nature of the diphtheria contagium, the supposition may reasonably be entertained, and supported by evidence, that the specific virus may be associated with some low form of micrococcus. From the persistency with which the infection hangs about particular spots - which my experience leads me to regard as the starting-points and foci of epidemics - it would appear not improbable that such an organism may survive and develop externally to, and independently of, the human body, spreading, perhaps, as a minute growth over congenial damp surfaces; nor would it be impossible that the influence 